\title{
Influence on interradicular bone volume of (1) CrossMark Invisalign treatment for adult crowding with interproximal enamel reduction: a retrospective three-dimensional cone-beam computed tomography study
}

\author{
Andreas Hellak ${ }^{1,3^{*}}$, Nicola Schmidt ${ }^{1}$, Michael Schauseil ${ }^{1}$, Steffen Stein ${ }^{1}$, Thomas Drechsler ${ }^{2}$ \\ and Heike Maria Korbmacher-Steiner ${ }^{1}$
}

\begin{abstract}
Background: The aim of this study was to use three-dimensional datasets to identify associations between treatment for adult crowding, using Invisalign aligner and interproximal enamel reduction (IER), and changes in the volume of interradicular bone.

Methods: A total of 60 cone-beam computed tomography (CBCT) scans from 30 adult patients (28 women, two men; 30 CBCTs pre-treatment, 30 post-treatment) were examined retrospectively in order to measure bone volume three-dimensionally. The patients' average age was $36.03 \pm 9.7$ years. The interradicular bone volume was measured with OsiriX at four levels in the anterior tooth areas of the maxilla and mandible. Differences in bone between T0 and T1 were analyzed with IBM SPSS 21.0 using the Wilcoxon test for paired samples.
\end{abstract}

Results: Overall, a slight increase in the quantity of bone was found $(0.12 \pm 0.73 \mathrm{~mm})$. There was a highly significant increase in bone in the mandible $(0.40 \pm 0.62 \mathrm{~mm} ; P<0.001)$, while in the maxilla there was a slight loss of bone, which was highly significant in the apical third $(-0.16 \pm 0.77 \mathrm{~mm} ; P=0.001)$.

Conclusions: Overall, treatment for adult crowding using an aligner and IER appears to have a positive effect on interradicular bone volume, particularly in patients with severe grades of the condition (periodontally high-risk dentition). This effect is apparently independent of IER. This is extremely important with regard to the treatment outcome, since IER and root proximity have been matters of debate in the literature and teeth should remain firmly embedded in their alveolar sockets.

Keywords: Aligner, Adult crowding, Interradicular bone volume, IER, Bone quantity, CBCT

\section{Background}

Among adult patients there is growing interest in having a functionally healthy and aesthetically attractive dentition [1]. Patients often have adult crowding and wish to have malpositioning corrected as invisibly as possible $[2,3]$.

\footnotetext{
* Correspondence: hellak@med.uni-marburg.de

${ }^{1}$ Department of Orthodontics, University Hospital Giessen and Marburg, Campus Marburg, Georg-Voigt-Strasse 3, 35039 Marburg, Germany

${ }^{3}$ Abt. für Kieferorthopädie, UKGM Standort Marburg, Georg-Voigt-Strasse 3, 35039 Marburg, Germany

Full list of author information is available at the end of the article
}

There are many treatment options e.g. using aligners and interproximal enamel reduction (IER).

One possible treatment for relieving crowding consists of expanding the dental arch in the labial direction in order to provide space for normal positioning of the affected teeth. Another method of creating space is IER. Potential periodontal changes in the anterior tooth area during orthodontic treatment with IER for adult crowding have been a topic of discussion in the literature [4-6]. In addition to the treatment of patients with periodontally healthy dentition, the question arises for the orthodontist

(c) The Author(s). 2018 Open Access This article is distributed under the terms of the Creative Commons Attribution 4.0 International License (http://creativecommons.org/licenses/by/4.0/), which permits unrestricted use, distribution, and reproduction in any medium, provided you give appropriate credit to the original author(s) and the source, provide a link to the Creative Commons license, and indicate if changes were made. The Creative Commons Public Domain Dedication waiver (http://creativecommons.org/publicdomain/zero/1.0/) applies to the data made available in this article, unless otherwise stated. 
of the way in which periodontally high-risk dentition is likely to behave during treatment. Vermylen et al. [7] defined an interradicular distance of $0.8 \mathrm{~mm}$ or less as root proximity and a risk marker for periodontal disease.

There have to date been no three-dimensional investigations of changes in interradicular bone volume in relation to treatment for adult crowding. The present study was carried out in order to investigate whether orthodontic treatment and resolution of crowding may even lead to an improvement in the bone situation. As a result of the use of conventional two-dimensional imaging to date, only limited quantification of the pre-therapeutic and post-therapeutic interradicular bone situation has been possible [8]. It is only modern three-dimensional cone-beam computed tomography $(\mathrm{CBCT})$ scanning that has made it possible to carry out $3 \mathrm{D}$ analysis of the bone structures and the way in which they respond to tooth movements $[9,10,11]$. The aim of the present study was to investigate whether and to what extent orthodontic treatment with Invisalign aligners and IER leads to a change in the interradicular bone volume. Specifically, the following questions were addressed:

- How is the interradicular bone volume altered by aligner therapy?

- What effects on interradicular root distances are associated with interproximal enamel reduction (IER)?

- In what ways does the interradicular bone volume change after initial findings corresponding to a root proximity (=interradicular distance of $\leq 0.8 \mathrm{~mm}$, a so called risk marker for periodontal disease [4])?

\section{Methods}

Changes in the interradicular distance were measured at a total of 720 measurement points in the present study. Pre-therapeutic and post-therapeutic cone-beam computed tomography (CBCT) scans from a total of 30 patients (28 women, two men) were examined retrospectively. In accordance with the SEDENTEXCT guidelines, the $\mathrm{CBCT}$ scans were taken for two reasons:

1. For periodontal assessment (a total of 26 cases). These patients had a fragile gingival type, with less bone in the anterior tooth area. Pre-treatment $\mathrm{CBCT}$ was therefore intended to visualize root proximity and resorption and help with therapeutic decision-making on whether to carry out IER or extraction of one anterior tooth. The following $\mathrm{CBCT}$ was intended to visualize root proximity and resorption after the completion of possible treatment, to check whether IER was still appropriate for creating space, since the teeth need to be covered by bone in order to avoid recession.
2. For temporomandibular joint assessment (a total of four cases). Some patients had craniomandibular disorders (CMD) with rheumatoid arthritis. In this small number of selected cases, pre-treatment $\mathrm{CBCT}$ was used to identify bone degenerative deformity, condylar positions, and bony structures in the temporomandibular joint. After successful CMD therapy and orthodontic treatment, CBCT scans were taken again to view the condylar positions and bony structures in the temporomandibular joint due to recurrent CMD problems and in order to adjust anti-inflammatory therapy.

The patients' average age was $36.03 \pm 9.7$ years. The use of the data was approved by the ethics committee of Marburg University Hospital (ref. no. 34/15).

The following inclusion and exclusion criteria were applied. Inclusion criteria:

- Presence of adult crowding capable of being adjusted using conservative orthodontic space-gaining measures such as protrusion, proclination, expansion and IER

- Permanent dentition

- Successfully completed treatment with Invisalign aligners

- Availability of one CBCT each from before and after treatment

The following parameters represented exclusion criteria:

- Extraction of anterior teeth during the course of treatment

- macrodontia/ hypoplasia

- abnormal change in tooth morphology

- Prosthetic treatment

- Skeletal anomalies

- General medical findings relevant to bone metabolism (e.g., osteoporosis, dysostosis, etc.)

- Periodontal disease and previous periodontal surgery procedures

All of the images were taken with a KaVo 3D eXam DVT system (KaVo Dental Ltd., Biberach an der Riss, Germany) using a scan with $360^{\circ}$ revolution, a duration of $26.9 \mathrm{~s}$ (X-ray source voltage: $120 \mathrm{kVp}$; X-ray source current: $5 \mathrm{~mA}$ ) and a voxel size of $0.25 \mathrm{~mm}$. The datasets were collected and evaluated using OsiriX (Pixmeo, Bernex, Switzerland) with an Apple OS X operating system.

All of the patients had provided written consent to the use of their data in the study (in accordance with the Helsinki Declaration). The data were all analyzed on a semi-blinded basis. 


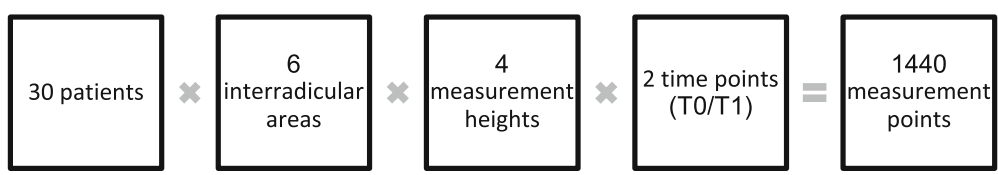

Fig. 1 Total measurement data

\section{Measurement of interradicular bone volume}

Measurements of the mesiodistal interradicular distance (Fig. 1) were modified in the OsiriX DICOM viewer using the method described by Sawada et al. [7]. The six interradicular areas between the lateral incisors in the maxilla and mandible were measured (Figs. 2 and 3).

The shorter of the roots in the two teeth adjoining the interradicular space was set as the reference tooth. A connecting line was drawn in the sagittal view from the buccal enamel-cement boundary (ECB) to the palatal or lingual enamel-cement boundary (Fig. 4). From the intersection of that line with the center of the root canal, the length to the apex was measured parallel to the dental axis/coronal plane (the ECB-apex distance), and the tooth was divided into four equal-sized sections. This resulted in the four measurement levels (Fig. 3). The sagittal view was used to adjust the axial plane to the desired height (Fig. 4).

The interradicular distance was measured in the axial view. For this purpose, two auxiliary lines were drawn parallel to the sagittal plane and were shifted in parallel as far as the root surfaces of the neighboring teeth (Fig. 5). In the measurement levels set, the interradicular distance was thus measured as the shortest distance between the root surfaces.

Whether and to what extent IER or expanding the dental arch in the labial direction influenced the interradicular space was investigated using ClinCheck software with the exact IER protocol. For each interradicular space we analyzed the change of the distance between the roots in comparison to the amount of IER. The total amount of IER was between $0.0 \mathrm{~mm}$ and $0.5 \mathrm{~mm}$. In our study crowding was defined as the difference in millimeter between the arch perimeter and the mesial to distal tooth size total form S1-S3 (upper anterior jaw) and S4-S6 (lower anterior jaw) (Fig. 2). Maxillary and mandibular arches were classified separately. Each case was classified as presenting mild discrepancy crowding between $0.1 \mathrm{~mm}$ to $-5 \mathrm{~mm}$ according to Proffit. W. R. and H. W. Field. Contemporary Orthodontics. St Louis, Mo: Mosby; 2000:224.

All of the digital volume tomograms were analyzed a second time by the same investigator (N.S.) one month later, to allow assessment of the reproducibility of the measurements. The means of the two evaluations were used for statistical analysis. All patients were treated by one operator (T.D.) in the same office.

Statistical analyses were carried out using IBM SPSS for Mac, version 21.0 (IBM Corporation, Armonk, New York, USA). The intraoperator correlation for each examination was initially calculated. For further analysis, the normal distribution of the values was checked. The values were tested for significant differences using the Wilcoxon test. The significance level was set at $P=0.05$.

\section{Results}

Kendall's tau-b test showed a highly significant $(P<0.001$, two-sided) intraoperator correlation $(r=0.837)$ for the interradicular distance measurements.

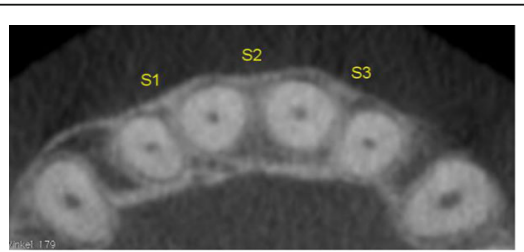

S1: space between 12 and 11

S2: space between 11 and 21

S3: space between 21 and 22

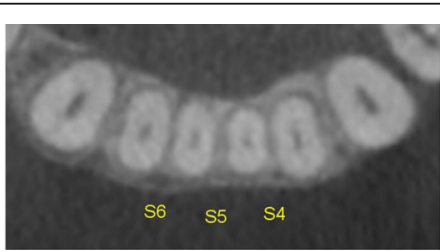

S4: space between 32 and 31

S5: space between 31 and 41

S6: space between 41 and 42

Fig. 2 Measurement points for interradicular distances 


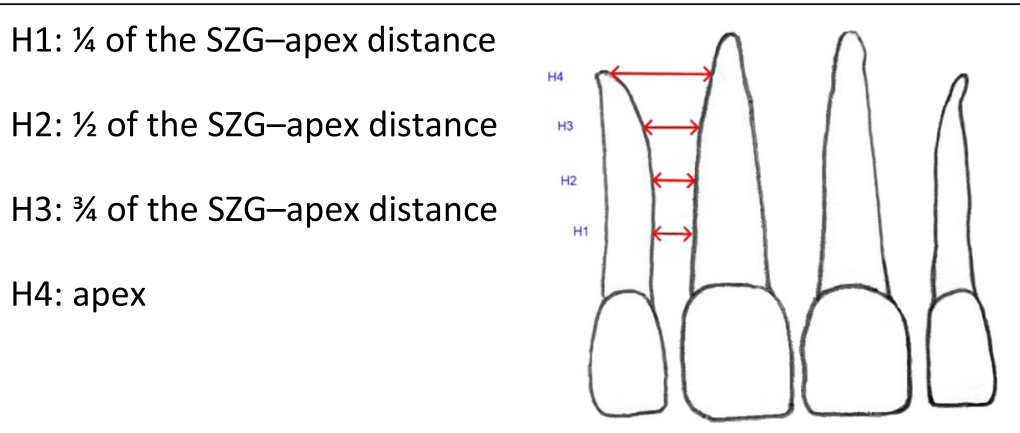

Fig. 3 Two-dimensional diagram showing the measurement distances used to determine interradicular distances

Increases in the interradicular distance were observed in the mandible, and decreases were observed in the maxilla (Table 1). The increase in interradicular space in the mandible was greater than the loss of space in the maxilla. The Wilcoxon test showed highly significant $(P \leq 0.001)$ changes between T0 and T1 at all levels in the mandible, highly significant $(P \leq 0.01)$ changes at the apex measurement level, and significant $(P \leq 0.05)$ changes at the three-quarter level in the maxilla (Table 1).

\section{Effects of IER}

A positive effect was noted after treatment in $62.5 \%$ of all interdental spaces in which IER was carried out; however, the distance decreased in $37.5 \%$. The effect was almost identical without IER (Table 2). Overall, it was found that IER did not have any statistically significant effects on the changing interradicular space conditions.

\section{Periodontally critical situation (interradicular distance $\leq 0.8 \mathrm{~mm}$ )}

In all, $17.2 \%$ of the pre-therapeutic interradicular measurement points had an interradicular distance $\leq 0.8 \mathrm{~mm}$ (Table 3), and the majority of these were in the mandible. As Table 3 shows, the treatment had a positive effect, since afterwards only $7.9 \%$ of the measurement points still had an interradicular distance $\leq 0.8 \mathrm{~mm}$.

It was then investigated whether a periodontally high-risk dentition $(\leq 0.8 \mathrm{~mm}$ ) benefited more from aligner treatment than a periodontally healthy dentition $(>0.8 \mathrm{~mm})$. Of the 124 measurement points that had a root distance $\leq 0.8 \mathrm{~mm}$ in the initial findings, $88.71 \%$ had increased space after treatment. An interradicular space increase of more than $0.8 \mathrm{~mm}$ was even observed in $71.77 \%$ (Table 4 ). An increase in space of more than $0.8 \mathrm{~mm}$ was observed in nine of 11 measurement points in the maxilla (81.82\%), and in 80 of 113 measurement points in the mandible (70.8\%).

By comparison, periodontally high-risk dentitions showed much larger increases in the interradicular bone volume (Table 5). As Table 5 shows, the result was highly significant statistically $(P \leq 0.001)$.

\section{Discussion}

In the group of patients investigated in the present study, treatment for adult crowding was associated with an overall increase in interradicular space. The increased space was gained particularly in the mandible, as the increase was larger than the slight loss of space in the maxilla. One possible explanation for this might be the

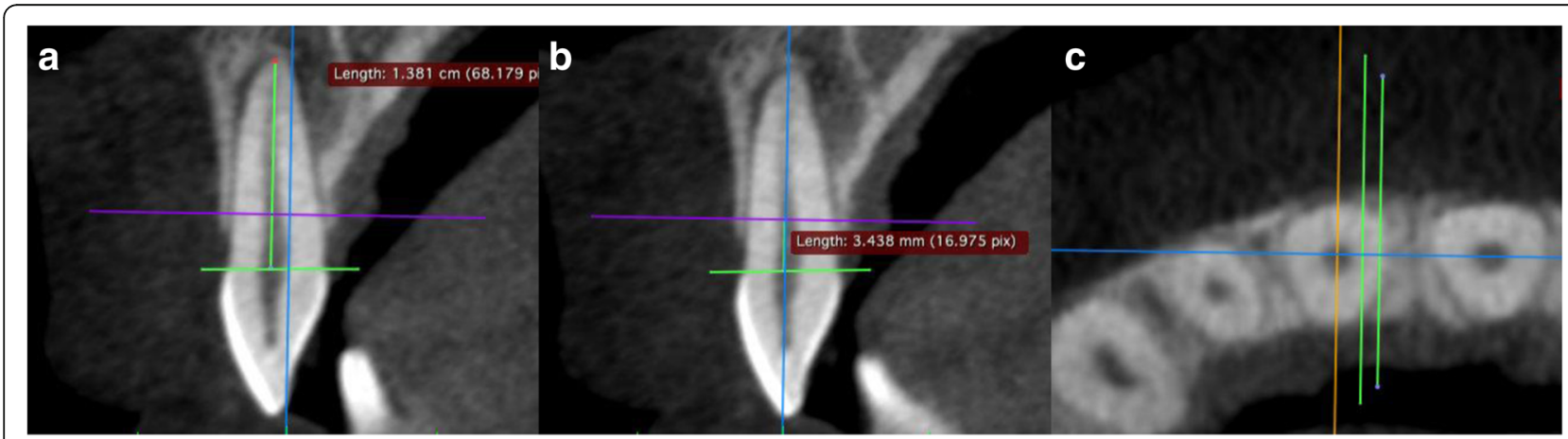

Fig. 4 Sagittal plane. a Measurement of the length of the apex to enamel-cement boundary (ECB) distance. b Setting the first measurement level, $\mathrm{H1}$, at one-quarter of the ECB-apex distance. c Creating the auxiliary lines parallel to the sagittal axis in the axial view 


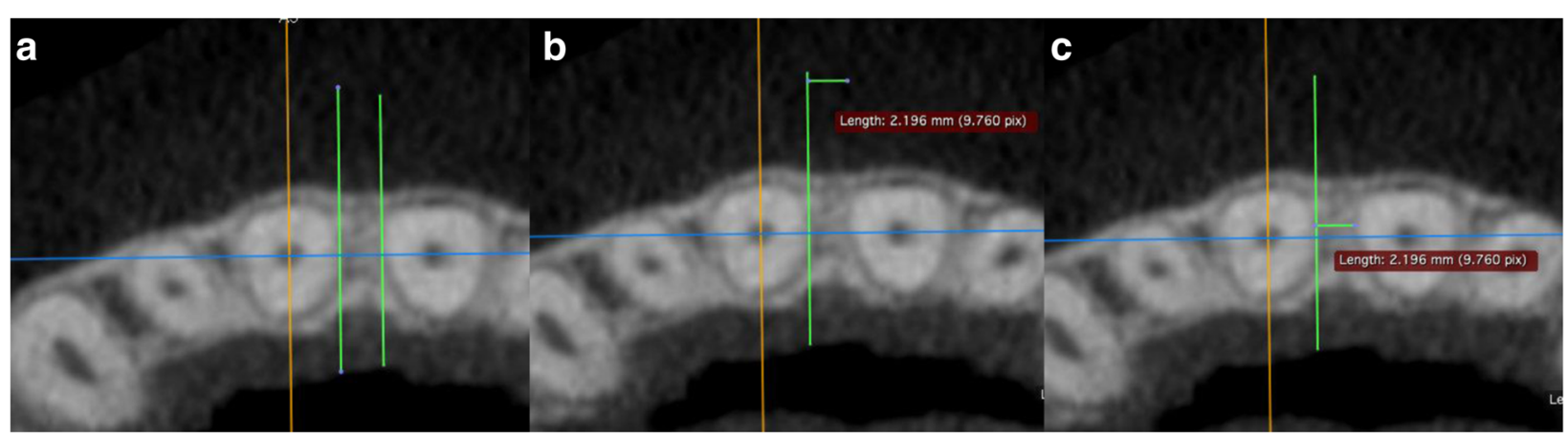

Fig. 5 Measurement of mesiodistal interradicular distance S1 at the $\mathrm{H} 1$ level on the axial plane. a Shifting of the auxiliary lines to the root surfaces. $\mathbf{b}$ Measuring the shortest interradicular distance by shifting an auxiliary line. c Display of the measurement distance

varying severity of the crowding. In this group of patients, adult crowding usually appeared earlier and with greater severity in the mandible than in the maxilla. More extensive measures to create space were therefore needed with a smaller bone volume. Another explanation might be the different methods used to create space.

One possible treatment for relieving crowding consists of expanding the dental arch in the labial direction in order to allow space for normal positioning of these teeth
[12]. This method of space creation was used much more often in the mandible. The positive effect of reshaping the dental arch thus appears to have a strong influence on increases in interradicular space.

Another method of creating space is IER [13]. Although the roots ought to move closer to each other after the removal of enamel during IER, the positive effect of reshaping the dental arch appears to outweigh this, at least in the mandible. This increase in space despite IER has

Table 1 Descriptive comparison of differences in the interradicular distance measurements between T1 and T0, using the Wilcoxon test $^{\mathrm{a}}$ for statistical analysis

\begin{tabular}{|c|c|c|c|c|c|c|c|c|}
\hline \multicolumn{2}{|c|}{ Measurement level } & $\mathrm{n}$ & Minimum & Maximum & Mean & SD & Wilcoxon test ${ }^{a}$ & T1-T0 \\
\hline \multicolumn{9}{|l|}{ Maxilla } \\
\hline \multirow[t]{2}{*}{$1 / 4$} & T1-T0 & 90 & -1.75 & 1.27 & -0.07 & 0.52 & Z & $-1.189^{b}$ \\
\hline & & & & & & & A. significance (two-sided) & 0.234 \\
\hline \multirow[t]{2}{*}{$1 / 2$} & T1-T0 & 90 & -2.40 & 1.33 & -0.03 & 0.66 & Z & $-0.127^{b}$ \\
\hline & & & & & & & A. significance (two-sided) & 0.899 \\
\hline \multirow[t]{2}{*}{$3 / 4$} & T1-T0 & 90 & -2.45 & 1.67 & -0.22 & 0.75 & z & $-2.505^{b}$ \\
\hline & & & & & & & A. significance (two-sided) & 0.012 \\
\hline \multirow[t]{2}{*}{ Apex } & $\mathrm{T} 1-\mathrm{T} 0$ & 90 & -3.02 & 1.94 & -0.32 & 1.03 & Z & $-2.565^{b}$ \\
\hline & & & & & & & A. significance (two-sided) & 0.01 \\
\hline \multicolumn{9}{|c|}{ Mandible } \\
\hline \multirow[t]{2}{*}{$1 / 4$} & T1-T0 & 90 & -0.68 & 1.35 & 0.30 & 0.46 & Z & $-5.237^{c}$ \\
\hline & & & & & & & A. significance (two-sided) & $<0.001$ \\
\hline \multirow[t]{2}{*}{$1 / 2$} & T1-T0 & 90 & -0.87 & 1.71 & 0.42 & 0.52 & Z & $-6.113^{c}$ \\
\hline & & & & & & & A. significance (two-sided) & $<0.001$ \\
\hline \multirow[t]{2}{*}{$3 / 4$} & T1-T0 & 90 & -1.33 & 2.49 & 0.45 & 0.62 & Z & $-6.051^{c}$ \\
\hline & & & & & & & A. significance (two-sided) & $<0.001$ \\
\hline \multirow[t]{2}{*}{ Apex } & T1-T0 & 90 & -1.86 & 2.60 & 0.40 & 0.84 & Z & $-4.048^{c}$ \\
\hline & & & & & & & A. significance (two-sided) & $<0.001$ \\
\hline
\end{tabular}


Table 2 Effects of interproximal enamel reduction on the interradicular distance in 180 interproximal spaces

\begin{tabular}{lll}
\hline & $\begin{array}{l}\text { With IER } \\
(n=104)\end{array}$ & $\begin{array}{l}\text { Without IER } \\
(n=76)\end{array}$ \\
\hline Interradicular distance increased & $62.50 \%$ & $63.16 \%$ \\
Interradicular distance decreased & $37.50 \%$ & $36.84 \%$ \\
\hline
\end{tabular}

IER interproximal enamel reduction

also been confirmed in other studies. In two-dimensional studies, Zachrisson et al. [4] found that due to crowding, the roots have to be closer together than in correctly aligned teeth.

During treatment for adult crowding, interproximal enamel reduction (IER) was only carried out supportively in a few interdental spaces. When only the subtopic of IER is considered, it is notable that IER did not have any significant effect on the bone volume between the anterior dental roots. The distribution pattern of changes in the interradicular distance was almost identical with and without IER.

In general, the advantage of interproximal enamel reduction is that the extent of the expansion in the labial direction can be reduced, thereby reducing the risk of bone dehiscence occurring. In addition, the widened approximal contacts stabilize the treatment result [14]. These findings are also consistent with those reported in the clinical studies by Zachrisson et al. [4], in which no deterioration was observed on dental film more than 10 years later after approximal enamel reduction. However, precise three-dimensional measurement of interradicular spatial conditions was not possible due to the use of two-dimensional radiographic diagnosis in the study. An improvement in the aesthetic appearance can also be expected as a result of relieving anterior crowding, due to the avoidance of what are known as "black triangles." The creation of optimal apposition areas for the gingiva also reduces or prevents retrusion of the interdental papillae [15].

The most noticeable positive effect was seen when teeth with root proximity were treated. Vermylen et al. [7] defined $0.8 \mathrm{~mm}$ or less bone or interdental tissue as representing root proximity. Interdental spaces of this size are poorly accessible for periodontal treatment and are less able to resist periodontal disease [16]. Radicular

Table 3 Interradicular distance $\leq 0.8 \mathrm{~mm}$ at time points T0 and $\mathrm{T} 1(n=720)$

\begin{tabular}{lll}
\hline & T0 & T1 \\
\hline Maxilla & $1.53 \%$ & $1.11 \%$ \\
Mandible & $15.69 \%$ & $6.81 \%$ \\
Total & $17.22 \%$ & $7.92 \%$ \\
\hline
\end{tabular}

Table 4 Increase in the interradicular distance between T0 and $\mathrm{T} 1$ of periodontally high-risk dentition, including interradicular space increases to $>0.8 \mathrm{~mm}$

\begin{tabular}{lll}
\hline$n=124(\leq 0.8 \mathrm{~mm})$ & Increases in space & Increases in space $>0.8 \mathrm{~mm}$ \\
\hline Maxilla & $90.91 \%$ & $81.82 \%$ \\
Mandible & $88.5 \%$ & $70.80 \%$ \\
Total & $88.71 \%$ & $71.77 \%$ \\
\hline
\end{tabular}

distances with this potentially poor initial condition showed improvement in the spatial situation in approximately $89 \%$ of cases, and improvement beyond the critical range (>0.8 $\mathrm{mm}$ after treatment) in approximately $72 \%$. This means that a periodontally high-risk dentition benefited more from the aligner treatment than a periodontally healthy dentition. However, it must be mentioned here that root proximity is not the cause of periodontal disease, but only represents a risk factor [17-20]. Bacterial plaque is one of the main causes of periodontal inflammation [21].

Another advantage of the present study is the three-dimensional imaging of the interradicular spaces. Other studies on measurement of bone volume have only been carried out using two-dimensional images, and have always noted the difficulty of depicting the interradicular distance precisely [8]. Two-dimensional images are also known as cumulative images. As a result of the cumulation, superimposed roots in crowded conditions are difficult to distinguish and the spaces are difficult to measure, due to differing enlargement factors. This lost information can be displayed in three-dimensional images [22]. For CBCT analysis, the question arises of whether the image resolution is sufficient to allow precise analysis. Gribel et al. [23] compared CBCT measurements with direct measurements of dry skulls. They found that CBCT scanning with a slice thickness of $0.3 \mathrm{~mm}$ was extremely precise, with a mean deviation of the measurements from the direct measurements of $0.1 \mathrm{~mm}$.

Unfortunaly this study has a retrospective design with a risk of bias. A prospective randomized controlled trial would be interesting, but could currently not be carried out because of the ALARA principle. In view of the principle that radiation exposure should be "as low as reasonably achievable" (ALARA), CBCT is not indicated as a routine method for the imaging of bone support $[24,25]$. A CBCT may only be indicated in selected cases in which clinical and conventional examinations do not provide the information needed for treatment. The operator always needs to consider its use carefully. If a CBCT is needed the use of shorter scans and a reduced effective radiation dose is recommended [26, 27]. A study group with a more balanced sex ratio would be desirable, because most of the patients included in this study were women. This is due to the retrospective 
Table 5 Descriptive statistics for interradicular changes, classified into groups with a root proximity (periodontally high-risk dentition - interradicular bone quantity at T0 $\leq 0.8 \mathrm{~mm}$ ) or with a periodontally normal dentition (interradicular bone volume at T0 > $0.8 \mathrm{~mm}$ ) with Wilcoxon signed rank test for statistical analysis

\begin{tabular}{lllllllll}
\hline Distance & & $\mathrm{n}$ & Min. & Max. & Mean & SD & Wilcoxon test $(\leq 0.8 \mathrm{~mm}$ vs. $>0.8 \mathrm{~mm})$ \\
\hline$\leq 0.8 \mathrm{~mm}$ & T1-T0 & 124 & -0.45 & 2.49 & 0.60 & 0.54 & Z & -8.071 \\
$>0.8 \mathrm{~mm}$ & T1-T0 & 596 & -3.02 & 2.60 & 0.02 & 0.75 & A. significance (two-sided) & $<0.001$ \\
\hline
\end{tabular}

SD standard deviation

character of our study. However, presenting these data from the context of aligner treatment for adult crowding and possible interradicular bone changes may be helpful. Further research and additional information on the topic would be desirable.

\section{Conclusions}

Overall, treatment of adult crowding using Invisalign and IER, particularly in patients with severe conditions (with periodontally high-risk dentition), appears to have a positive effect on the interradicular bone volume, at least in adult female patients. The effect is also apparently independent of IER.

\section{Abbreviations}

ALARA: As low as reasonably achievable; CBCT: Cone beam computed tomography scans; IER: Interproximal enamel reduction

\section{Acknowledgments}

The authors thank Mr. Robertson for editing the manuscript.

\section{Funding}

The present study was not funded, nor supported by any grant. Therefore, the authors report no conflict of interest related to the present work.

\section{Availability of data and materials}

We state that the clinical data and personal details will not be made available in order to protect the participants identity.

\section{Authors' contributions}

$\mathrm{AH}$ conceived the study together with NS and HMKS, and carried out all experiments and drafted the manuscript. AH and MS performed the statistical analysis. TD treated the patients and SS helped with the collection of the data and made substantial contributions to conception and design. HMKS conceived the study, participated in its design and coordination and helped to draft the manuscript. All authors read and approved the final manuscript.

\section{Ethics approval and consent to participate}

All research activities relevant to the present study were performed in accordance with the Declaration of Helsinki. This study involves human data, and the use of the data was approved by the ethics committee of Marburg University Hospital (ref. no. 34/15). All of the patients had provided written consent to the use of their data in the study.

\section{Competing interests}

The authors declare that they have no competing interests.

\section{Publisher's Note}

Springer Nature remains neutral with regard to jurisdictional claims in published maps and institutional affiliations.

\section{Author details}

${ }^{1}$ Department of Orthodontics, University Hospital Giessen and Marburg Campus Marburg, Georg-Voigt-Strasse 3, 35039 Marburg, Germany. ${ }^{2}$ Private practice, Wiesbaden, Germany. ${ }^{3}$ Abt. für Kieferorthopädie, UKGM Standort Marburg, Georg-Voigt-Strasse 3, 35039 Marburg, Germany.

Received: 8 December 2017 Accepted: 31 May 2018

Published online: 08 June 2018

\section{References}

1. Nedwed V, Miethke RR. Motivation, acceptance and problems of Invisalign patients. J Orofac Orthop. 2005;66(2):162-73.

2. Weir T. Clear aligners in orthodontic treatment. Aust Dent J. 2017:62(1):58-62.

3. Meier B, Wiemer KB, Miethke RR. Invisalign-patient profiling. Analysis of a prospective survey. J Orofac Orthop. 2003:64(5):352-8.

4. Zachrisson BU, Nyøygaard L, Mobarak K. Dental health assessed more than 10 years after interproximal enamel reduction of mandibular anterior teeth. Am J Orthod Dentofac Orthop. 2007;131(2):162-9.

5. Zachrisson BU, Minster L, Ogaard B, Birkhed D. Dental health assessed after interproximal enamel reduction: caries risk in posterior teeth. Am J Orthod Dentofac Orthop. 2011;139(1):90-8.

6. Renkema AM, Navratilova Z, Mazurova K, Katsaros C, Fudalej PS. Gingival labial recessions and the post-treatment proclination of mandibular incisors. Eur J Orthod. 2015:37(5):508-13.

7. Vermylen K, De Quincey GN, van 't Hof MA, Wolffe GN, Renggli HH. Classification, reproducibility and prevalence of root proximity in periodontal patients. J Clin Periodontol. 2005;32(3):254-9.

8. Zachrisson BU, Alnaes L. Periodontal condition in orthodontically treated and untreated individuals. II. Alveolar bone loss: radiographic findings. Angle Orthod. 1974;44(1):48-55.

9. Fuhrmann R. Three-dimensional interpretation of periodontal lesions and remodeling during orthodontic treatment. Part III. J Orofac Orthop. 1996; 57(4):224-37.

10. Lund $\mathrm{H}$. Cone beam computed tomography in evaluations of some side effects of orthodontic treatment. Swed Dent J Suppl. 2011:219:4-78.

11. Sawada K, Nakahara K, Matsunaga S, Abe S, Ide Y. Evaluation of cortical bone thickness and root proximity at maxillary interradicular sites for miniimplant placement. Clin Oral Implants Res. 2013;24 Suppl A100:1-7.

12. Stroud JL, English J, Buschang PH. Enamel thickness of the posterior dentition: its implications for nonextraction treatment. Angle Orthod. 1998; 68(2):141-6.

13. Sheridan JJ. Air-rotor stripping. J Clin Orthod. 1985;19(1):43-59.

14. Allais D, Melsen B. Does labial movement of lower incisors influence the level of the gingival margin? A case-control study of adult orthodontic patients. Eur J Orthod. 2003;25(4):343-52.

15. Melsen B, Allais D. Factors of importance for the development of dehiscences during labial movement of mandibular incisors: a retrospective study of adult orthodontic patients. Am J Orthod Dentofac Orthop. 2005: 127(5):552-61. quiz 625

16. Kim T, Miyamoto T, Nunn ME, Garcia Rl, Dietrich T. Root proximity as a risk factor for progression of alveolar bone loss: the veterans affairs dental longitudinal study. J Periodontol. 2008;79(4):654-9.

17. Bollen AM. Effects of malocclusions and orthodontics on periodontal health: evidence from a systematic review. J Dent Educ. 2008;72(8):912-8.

18. Uysal T, Yagci A, Ozer T, Veli I, Ozturk A. Mandibular anterior bony support and incisor crowding: is there a relationship? Am J Orthod Dentofac Orthop. 2012:142(5):645-53.

19. Alsulaiman AA, Kaye E, Jones J, Cabral H, Leone C, Will L, Garcia R. Incisor malalignment and the risk of periodontal disease progression. Am J Orthod Dentofac Orthop. 2018;153(4):512-22.

20. Diedrich P. Periodontal relevance of anterior crowding. J Orofac Orthop. 2000;61(2):69-79. 
21. Corbet EF, Davies WI. The role of supragingival plaque in the control of progressive periodontal disease. A review. J Clin Periodontol. 1993;20(5): 307-13.

22. Schulze D, Fleiner J. Digitale dentale Volumentomografie. Zahnmed Up2date. 2008;2:247-59.

23. Gribel BF, Gribel MN, Frazäo DC, McNamara JA Jr, Manzi FR. Accuracy and reliability of craniometric measurements on lateral cephalometry and $3 \mathrm{D}$ measurements on CBCT scans. Angle Orthod. 2011;81(1):26-35.

24. Ludlow JB, Davies-Ludlow LE, Brooks SL, Howerton WB. Dosimetry of 3 CBCT devices for oral and maxillofacial radiology: CB Mercuray, NewTom $3 G$ and i-CAT. Dentomaxillofac Radiol. 2006;35(4):219-26. Erratum in: Dentomaxillofac Radiol 2006;35(5):392

25. Silva MA, Wolf U, Heinicke F, Bumann A, Visser H, Hirsch E. Cone-beam computed tomography for routine orthodontic treatment planning: a radiation dose evaluation. Am J Orthod Dentofac Orthop. 2008:133(5):640. e1-5

26. Cook VC, Timock AM, Crowe JJ, Wang M, Covell DA Jr. Accuracy of alveolar bone measurements from cone beam computed tomography acquired using varying settings. Orthod Craniofac Res. 2015;18(Suppl 1):127-36.

27. Padala S, Tee BC, Beck FM, Elias K, Kim DG, Sun Z. The usefulness of cone-beam computed tomography gray values for alveolar bone linear measurements. Angle Orthod. 2018;88(2):227-32.

Ready to submit your research? Choose BMC and benefit from:

- fast, convenient online submission

- thorough peer review by experienced researchers in your field

- rapid publication on acceptance

- support for research data, including large and complex data types

- gold Open Access which fosters wider collaboration and increased citations

- maximum visibility for your research: over $100 \mathrm{M}$ website views per year

At BMC, research is always in progress.

Learn more biomedcentral.com/submissions 